\title{
A functionalized poly(ethylene glycol)-based bioassay surface chemistry that facilitates bio-immobilization and inhibits non- specific protein, bacterial, and mammalian cell adhesion
}

\author{
Gregory M. Harbers ${ }^{1,3}$, Kazunori Emoto ${ }^{2}$, Charles Greef $^{2}$, Steven W. Metzger ${ }^{2}$, Heather N. \\ Woodward $^{2}$, James J. Mascali ${ }^{2}$, David W. Grainger ${ }^{1,4}$, and Michael J. Lochhead ${ }^{2, *}$ \\ 1 Department of Chemistry, Colorado State University, Fort Collins, CO 80523-1872 USA \\ 2 Accelr8 Technology Corporation, 7000 N. Broadway, Suite 3-307, Denver, CO 80221 USA
}

\begin{abstract}
This paper describes a new bioassay surface chemistry that effectively inhibits non-specific biomolecular and cell binding interactions, while providing a capacity for specific immobilization of desired biomolecules. Poly(ethylene glycol) (PEG) as the primary component in nonfouling film chemistry is well-established, but the multicomponent formulation described here is unique in that it (1) is applied in a single, reproducible, solution-based coating step; (2) can be applied to diverse substrate materials without the use of special primers; and (3) is readily functionalized to provide specific attachment chemistries. Surface analysis data are presented, detailing surface roughness, polymer film thickness, and film chemistry. Protein non-specific binding assays demonstrate significant inhibition of serum, fibrinogen, and lysozyme adsorption to coated glass, indium tin oxide, and tissue culture polystyrene dishes. Inhibition of S. aureus and K. pneumoniae microbial adhesion in a microfluidic flow cell, and inhibition of fibroblast cell adhesion from serum-based cell culture is shown. Effective functionalization of the coating is demonstrated by directing fibroblast adhesion to polymer surfaces activated with an RGD peptide. Batch-to-batch reproducibility data are included. The in situ cross-linked PEG-based coating chemistry is unique in its formulation, and its surface properties are attractive for a broad range of in vitro bioassay applications.
\end{abstract}

\section{INTRODUCTION}

Inhibition of non-specific biomolecular and cellular adhesion to solid surfaces is critical to the performance of in vitro bioassays. Protein non-specific binding (NSB), for example, is long recognized as limiting the sensitivity of solid-phase assays such as immunoassays and microarrays ${ }^{1,2}$. Further, the emergence of analytical tools in which live, phenotypically correct cells are maintained in culture milieu at synthetic surfaces has created new surface chemistry challenges ${ }^{3}$. Improved surface treatments and coating technologies that provide better nonfouling performance in conjunction with specific attachment chemistries are sought for these applications.

The use of poly(ethylene glycol) (PEG) polymers and PEG-like materials as the active component in nonfouling surface chemistries is well-established and the subject of several

\footnotetext{
*To whom correspondence should be addressed. Accelr8 Technology Corporation, 7000 N. Broadway, Suite 3-307, Denver, CO 80221. EMAIL: mlochhead@accelr8.com; PHONE: 303-863-8088; FAX: 303-863-1218.

${ }^{3}$ Current address: Affinergy, Inc., 617 Davis Dr., Suite 100, Durham, NC 27713 USA

${ }^{4}$ Current address: Department of Pharmaceutics and Pharmaceutical Chemistry, College of Pharmacy, 30 South 2000 East, Room 301, Salt Lake City, UT 84112-5820 USA
} 
reviews $4-7$. A variety of surface treatment methods have been reported, including PEG grafting $6,8-18$, adsorptive chemistries ${ }^{19-24}$, self-assembled monolayers (SAMs) ${ }^{25-27}$, and plasma treatments ${ }^{28}$. Outstanding protein and cellular non-specific binding inhibition has been demonstrated in several of these systems.

Despite significant progress, however, many of these surface chemistries suffer from one or more limitations that hinder widespread application. For example, grafting and SAM technologies are typically compatible only with specific, well-defined substrates. Passively adsorbed coating chemistries typically require a charged or hydrophobic surface and can desorb under stringent wash conditions. Many coating methods require multiple, difficult-to-control processing steps to achieve a high quality final film. Robust, readily functionalized PEG-based thin films applicable to a variety of substrates and amenable to scalable manufacturing are not widely known.

The multicomponent, cross-linked polymer surface chemistry described here was designed to address several of these limitations. The single-step coating formulation which can be applied with conventional industrial processing techniques, was developed specifically for in vitro bioassay applications, and combines covalent surface attachment and cross-linking chemistries within the coating matrix. Significant inhibition of protein, microbial and mammalian cell adsorption and adhesion on a variety of relevant substrate materials is demonstrated, while selective functionalization of the coating is shown using specific coupling of an RGD peptide that enables cell attachment in a standard cell culture format. Batch-to-batch specific and nonspecific binding reproducibility data are also presented.

\section{EXPERIMENTAL SECTION}

\section{Materials}

Substrates consisted of borosilicate glass microscope slides (Schott Glass, D263, 75.6 25.0 $\times 1.0 \mathrm{~mm}$ ), tissue culture polystyrene (TCPS) Petri dishes (BD Falcon), indium tin oxide (ITO) coated glass slides (Delta Technologies, $75 \times 25 \times 1.1 \mathrm{~mm}$ ), and $\mathrm{SiO}_{2} / \mathrm{Si}$ wafers (Montco Silicon, $125 \mathrm{~mm}$ dia. $\times 0.5 \mathrm{~mm}$ thick). Homobifunctional polyethylene glycol (PEG, mol. wt. 3400), with N-oxy-succinimide termini on each end of the PEG molecule, was from NOF, Japan. Here we use the common name NHS-PEG-NHS for this molecule, where NHS is the $\mathrm{N}$-hydroxy-succinimide leaving group. Additional coating reagents included organosilanes (3trimethoxysilylpropyl) diethylenetriamine ("aminosilane") and 6-azidosulfonylhexyltriethoxy silane, ("azidosilane") (Gelest, Inc.), polyoxyethylene sorbitan tetraoleate (PST), methoxyethylamine (MEA), and solvents methyl sulfoxide (DMSO) and N,Ndimethylacetamide (DMAc) (reagent grade, used as received, all from Sigma-Aldrich). Goat serum, human fibrinogen ( $80 \%$ clottable), and hen egg white lysozyme were from SigmaAldrich. Horseradish peroxidase (HRP)-conjugated goat anti-human fibrinogen and rabbit antilysozyme IgG's were from Abcam, Inc. Goat anti-rabbit IgG and rabbit anti-goat IgG, each labeled with the fluorescent dye Alexa555, were from Molecular Probes, Inc, as was the NHSAlexa555 used for protein labeling. GRGDS was from American Peptide Company, Inc. Buffer salts, bovine serum albumin (BSA, Fraction V), and other laboratory reagents were obtained from Sigma-Aldrich. The acronym PBS designates phosphate buffered saline $(0.1 \mathrm{M}$ sodium phosphate, $0.15 \mathrm{M}$ sodium chloride, $\mathrm{pH}$ 7.2). PBST is PBS that contains $0.05 \%$ Tween $20^{\circledR}$. Custom 40mer oligonucleotide probes were from Integrated DNA Technologies (IDT, Coralville, IA). Unmodified and amine-modified probes had identical sequences. Complementary 40mer target sequences with and without terminal Alexa555 labels were also from IDT. 


\section{Surface Chemistry and Coating Process}

Substrate Cleaning-Glass and ITO/glass slides were sonicated at $60^{\circ} \mathrm{C}$ in alkaline detergent, extensively water rinsed, racked, dried by centrifugation, and stored in a cleanroom drybox until coating (within 24 hours of cleaning). $\mathrm{SiO}_{2} / \mathrm{Si}$ wafers were cleaned using a Harrick Plasma cleaner (5 minutes with argon plasma). TCPS dishes were coated as received. Importantly, all clean substrates were used as-is; no silane treatments or other bonding promoters were used.

Coating Formulation-The coating formulation comprises a single mixture of three components described here as (1) the active component, (2) matrix-forming component, and (3) cross-linking component, all mixed in a carrier solvent ${ }^{29}$. The coating components and process are illustrated schematically in Figure 1. The active component is a heterobifunctional PEG molecule (A-PEG-B) with one end group (A) that serves as the functional group in the final coating. The other PEG end group (B), the reactive group, provides covalent attachment within the coating matrix and to certain substrates. PEGs in the molecular weight range of 2000 to 5000 have been successfully used. In the formulation described here, the active component was a NHS-PEG-aminosilane construct formed by reacting NHS-PEG-NHS (MW 3400) with (3-trimethoxysilylpropyl) diethylenetriamine (aminosilane) at a 2:1 mole ratio. The A-PEG-B hetero-construct is the desired reaction product. Incomplete reactions and competing reactions likely produce a mixture of reaction products that include A-PEG-B, B-PEG-B, and A-PEGA. Optimization of reactant concentrations, reaction times, and temperatures is discussed in more detail below.

The matrix-forming component was a non-ionic surfactant containing ethylene oxide repeat units. Polyoxyethylene sorbitan oleates such as the Tween ${ }^{\circledR}$ family of surfactants have been successfully used. Here, polyoxyethylene sorbitan tetraoleate (PST) served as the matrixforming component.

Azidosilane was used as a molecular cross-linker ${ }^{30}$. Upon thermal activation, the azide converts to a reactive nitrene that rapidly and non-specifically inserts into aliphatic or aromatic bonds within the coating matrix and into organic surfaces such as polystyrene ${ }^{31}$. Additionally, the silane chemistry reacts both with oxide solid surfaces and with the aminosilane component in the mixture.

Conceptually, the coating formulation is designed to provide multiple reaction pathways for converting the soluble three-component mixture to a stable, resilient functional coating through several possible cross-linking, adhesion, and covalent attachment mechanisms upon curing. In contrast to previous PEG brush technologies that typically rely on end point attachment of polymer chains to the surface or adsorptive chemistries that rely on relatively weak interactions, the intent here is to provide multiple attachment and covalent cross-linking modalities within a single formulation. The ampiphilic matrix forming component is needed to improve the ability to process the coating formulation since removal of this component results in final films that are highly phase-separated and of exceptionally low-quality. It has previously been demonstrated that all components are necessary for the formation of the final film ${ }^{29}$.

When engineering the coating formulation, relative reagent concentrations were optimized through extensive functional testing of the final film (contact angle, NHS activity, protein nonspecific binding, etc.), rather than analytical characterization of all intermediate reaction products which, although scientifically interesting, was deemed unnecessary since the end goal was to develop a single formulation that was easily applied and resulted in a robust, aminereactive, low non-specific binding film useful for in vitro diagnostics. For example, the NHSPEG to aminosilane ratio was optimized to provide reproducible film uniformity while maximizing NHS activity in the final film. The azidosilane cross-linker concentration was 
optimized to yield a reproducible film surface energy as determined by water contact angle. Removing the azidosilane altogether yielded films with highly variable surface energy that were less stable under stringent wash conditions. Additionally, the azide groups also provide the means for covalent attachment to plastic substrates. Thus, while the formulation may appear to be complex, the reagents, coating process, and curing conditions have been optimized to render a final coating that is relatively insensitive to incompletely characterized intermediate reactions.

The formulation is described in detail here. NHS-PEG-NHS (MW 3400) was mixed with (3trimethoxysilylpropyl) diethylenetriamine (aminosilane) at a 2:1 mole ratio in DMAc in a polypropylene conical tube. The reactant concentrations were $19.88 \mathrm{mM}$ NHS-PEG-NHS and $9.89 \mathrm{mM}$ aminosilane, respectively. These were allowed to react at ambient temperature (21 to $23^{\circ} \mathrm{C}$ ) with stirring for two hours. In a separate conical tube, a mixture in DMSO was prepared containing $8.26 \mathrm{mM}$ 6-azidosulfonylhexyltriethoxy silane and $2.17 \mathrm{mM}$ PST. After the two hour reaction, the DMAc-based and DMSO-based components were mixed in a 1 to 5.59 volume ratio with gentle stirring, and additional DMSO was added, yielding final formulation concentrations of $2.52 \mathrm{mM}$ NHS-PEG-NHS, $1.25 \mathrm{mM}$ aminosilane, $5.84 \mathrm{mM}$ azidosilane, and $1.53 \mathrm{mM}$ PST.

This formulation is stable at ambient temperature $\left(21\right.$ to $23^{\circ} \mathrm{C}$ ) for several days. When stored longer, formulation viscosity increases, likely resulting from silane hydrolysis and condensation. This oligomerization of the coating components results in increased final film thickness. In order to ensure consistent thickness, all coatings described in this paper were deposited within 48 hours of formulation.

Spin Coating, Cure, and Rinse-Solution spin coating used conventional bench top spinners (Laurell Technologies) at $3500 \mathrm{rpm}$ under ambient ventilated conditions unless indicated otherwise. After spinning, the coating was thermally cured at $0.1 \mathrm{~mm} \mathrm{Hg}$ pressure to drive the cross-linking reaction within the film and to remove solvent. Coatings on inorganic substrates were cured for 75 minutes at $100^{\circ} \mathrm{C}$. For the TCPS, the curing temperature was reduced to $70^{\circ} \mathrm{C}$ to avoid substrate softening. After cure, all surfaces were rinsed briefly with ultrapure water to remove any loosely bound material, and immediately dried in ambient air in a centrifuge using slide racks in a microplate swing holder. Coated substrates were stored dry in sealed moisture barrier bags with desiccant.

Surface Deactivation-The coating chemistry is designed to provide an NHS-activated polymer coating after cure. Prior to protein non-specific binding and bacterial adhesion assays, reactive NHS groups were quenched by submerging slides in $50 \mathrm{mM}$ methoxyethylamine (MEA) in $50 \mathrm{mM}$ borate buffer ( $\mathrm{pH} 9.0$ ) for one hour at room temperature. The MEA amine terminus reacts with the NHS end groups to create amide-linked ethyl methoxy groups terminating linear PEG chains. For the mammalian cell adhesion assays, NHS groups were hydrolyzed in buffer, resulting in carboxylate-terminated linear PEG chains.

Safety Consideration: All chemical formulation steps and spin operations were performed in certified chemical fume hoods by trained personnel wearing proper personal protective equipment.

\section{Surface Analysis}

Atomic Force Microscopy (AFM)_AFM measurements were performed on a Digital Instruments NanoScope IIIa. Silicon tips (Nanosensors; resonant frequency of 75 to $90 \mathrm{~Hz}$; force constant of 2.5 to $4.4 \mathrm{~N} / \mathrm{m}$ ) were used in tapping mode with a scan rate of $1.00 \mathrm{~Hz}$. Samples were measured both dry ( $40 \%$ relative humidity) and under water. 
Spectroscopic Ellipsometry-Spectroscopic ellipsometry was used to determine dry polymer film thickness on silicon substrates. All data were collected at room temperature $\left(\sim 22^{\circ}\right.$ C) and relative humidity ( $40 \%$ ) using a Woollam VASE VB-250 system equipped with a HS-190 monochromator and controlled by Wvase32 software (Woollam, v. 3.398c). For each substrate, data were collected at three incident angles $\left(65^{\circ}, 70^{\circ}\right.$, and $\left.75^{\circ}\right)$ from 300 to $1100 \mathrm{~nm}$. Experimental Cauchy fits to the spectroscopic data were performed using the Wvase 32 software.

X-Ray Photoelectron Spectroscopy (XPS) - XPS surface analyses were performed as recently described ${ }^{32}$ on a Physical Electronics PE5800 ESCA/AES system equipped with a 7 $\mathrm{mm}$ monochromatic $\mathrm{Al} \mathrm{K} \alpha \mathrm{X}$-ray source $(1486.6 \mathrm{eV})$ and hemispherical analyzer at a $35^{\circ}$ photoelectron take-off angle, defined as the angle between the surface plane and the axis of the analyzer lens. At this angle, sampling depth averages $\sim 5 \mathrm{~nm}$.

Aqueous Contact Angle (wetting) - All contact angles were measured at ambient conditions $\left(\sim 22^{\circ} \mathrm{C} ; 30\right.$ to $40 \%$ rel. humidity) using ultrapure water (ASTM type I water, $18.2 \mathrm{M} \Omega \bullet \mathrm{cm}$ ). Static water contact angle measurements were performed using a custom-built video droplet analysis instrument. Dynamic water contact angles (advancing and receding) were measured using a Krüss drop shape analysis system DSA-10 fitted with a NE43 needle (Krüss USA, Matthews, NC).

\section{Biological Assay Methods}

Protein Non-Specific Binding (NSB)—Goat serum was selected as a model system because it mimics a critical assay fluid for in vitro diagnostic and other bioassay formats. Fibrinogen was used because it is a large $(340 \mathrm{kD})$ glycoprotein central to blood clotting, and widely used as a model surface-active protein in non-specific binding assays ${ }^{33}$. Lysozyme $(14 \mathrm{kD})$ was selected because of its unique basic protein character (pI 10.7); lysozyme carries a positive charge at or near neutral $\mathrm{pH}^{34}$ and has also been widely used as a model NSB protein $^{27,35}$.

Details of the protein NSB immunoassays, including incubation methods, times, and temperatures, are provided in the Supporting Information. Briefly, goat serum was labeled with the fluorescent dye NHS-Alexa555 (Molecular Probes) using vendor protocols, and total protein concentration was determined by BCA assay (Pierce Biotechnology, Inc). Final protein concentration was adjusted to $10 \%$ total protein of the original, unlabeled serum by diluting with PBS. NSB assays were performed with $10 \%, 1 \%$, and $0.1 \%$ serum (in PBS), corresponding to total protein concentrations of $7.3,0.73$, and $0.073 \mathrm{mg} / \mathrm{ml}$, respectively.

Lysozyme and fibrinogen were assayed in an antibody half-sandwich format. Briefly, assays were performed using standard ELISA-like methods at room temperature with one hour incubations and PBST rinses. Surface-bound fluorescence signal was measured using a Tecan LS-400 laser scanner.

Due to fluorescence quenching on ITO and high TCPS autofluorescence during laser scanning, enzyme immunoassays were performed on these surfaces. After incubation with the horseradish peroxidase (HRP) labeled secondary antibody, surfaces were incubated with a colorimetric peroxidase substrate (SureBlue, 1-Component TMB, KPL, Inc.) and optical density was read at $450 \mathrm{~nm}$ using a plate reader (Dynex Opsys MR).

Control surfaces for both the fluorescence and enzyme assays were bare and BSA-blocked substrates. BSA blocking was performed by incubating the surface with 5\% BSA in PBS for one hour at room temperature, followed by a PBST then water rinse. 
Previous studies have provided reasonable correlation between fluorescence and enzymebased assays such as those performed here with more quantitative non-specific binding assays 36,37 .

Bacterial Adhesion-Bacterial retention on surfaces was assessed using a custom-built microfluidic device designed to provide well-controlled hydrodynamic conditions ${ }^{38}$. The device comprises plastic laminate assemblies (Aline, Inc.) defining flow channels sandwiched between two transparent coated slides (glass or ITO). Eight independent flow channels, each $10 \mathrm{~mm}$ long, $1.7 \mathrm{~mm}$ wide and $0.275 \mathrm{~mm}$ deep (rectangular cross section) were observed in real time using darkfield microscopy on an inverted microscope (Olympus IX-70) equipped with a CCD camera (MicroFire). The inlet and outlet ports of each flow channel were fitted with PEEK tubing connected to a custom-built pump station actuating a syringe pump (Kloehn) providing controlled flow delivery in a range from 0.085 to 5 microliters $/ \mathrm{sec}$. These flow rates and channel dimensions result in wall shear rates of 4.6 to $270 \mathrm{sec}^{-1}$. Two model bacterial strains were used. Staphylococcus aureus (ATCC BAA-977) served as model Gram-positive cocci. Klebsiella pneumoniae (ATCC 49472) served as model gram-negative rods. These are clinically relevant, pneumonia-causing microorganisms. Fresh (3 hour, sub-cultured in tryptic soy broth) cell cultures were centrifuged, re-suspended in PBS at a concentration of $3 \times 10^{7}$ colony forming units (CFU) per ml. Approximately 500 microliters of this solution was injected through the flow cell, then cells settled and incubated at room temperature with the basal test surface in the absence of flow for one hour. Images were captured after settling to establish surface baseline cell counts, which were approximately $5 \times 10^{4}$ cells $/ \mathrm{cm}^{2}$. After the static incubation, controlled flow was then initiated and surface images collected for wall shear rates of 4.6, 27, and $270 \mathrm{sec}^{-1}$. Five imaged fields of view were collected for each condition and custom image analysis software was used to generate adherent bacterial counts. These conditions mimic fluid flow encountered in microfluidic cell culture devices and therefore provide a suitable model ${ }^{39}$.

Safety Consideration: The bacterial strains used here are class II human pathogens. All bacterial assays were performed in a Biosafety Level II Laboratory by trained personnel with proper containment equipment.

Mammalian Cell Adhesion-NIH 3T3 fibroblast (ATCC, MD) adhesion assays were performed on inert polymer-coated surfaces as well as RGD-functionalized polymer surfaces. The first condition was used to demonstrate inhibition of mammalian cell adhesion, while the second demonstrated that the coating can be modified to provide specific functionality, in this case enabling cell attachment via the well-known cell-binding domain, arginine-gylcineaspartic acid (RGD) ${ }^{40-43}$.

NHS-activated polymer-coated glass slide pieces $(8 \times 8 \mathrm{~mm})$ were either $(1)$ immersed in

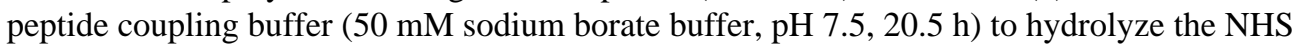
ester to carboxyl groups, or (2) incubated in peptide coupling buffer containing the cell-binding peptide, 100 micromolar GRGDS, for $20.5 \mathrm{~h}$ in 24 -well plates at ambient temperature (1 sample/well; 500 microliter solution/well). Tissue culture polystyrene (TCPS) was used as the positive control cell-adhesive surface.

Cell Seeding and Measurement: NIH 3 T3 fibroblasts (passage 23; ATCC) were maintained using established methods ${ }^{44}$. Details of the cell seeding and measurement are provided in the Supporting Information. Briefly, samples were seeded at $15000 \mathrm{cells} / \mathrm{cm}^{2}$ (30000 cells/well; 24-well Falcon TCPS plate) and incubated $\left(37^{\circ} \mathrm{C}, 5 \% \mathrm{CO}_{2}\right)$ for 8 and $24 \mathrm{~h}$ in growth media (GM) containing $10 \%$ serum. At specified times ( 8 and $24 \mathrm{~h}$ ), samples were aspirated, rinsed $1 \mathrm{x}$ with PBS (1 mL; HyQ) at ambient temperature, replenished with $\mathrm{GM}$, and imaged using 
an inverted Nikon TE2000-U microscope equipped with a Coolsnap ES CCD camera driven by Metamorph software (Universal Imaging Corp).

Reproducibility Assays-In order to assess coating formulation and process reproducibility, three coated glass slide production batches prepared on three different days over a 5 week period were assayed in parallel. Each production batch comprised 300 to 450 slides, and three slides from each batch were selected to represent the beginning, middle, and end of the coating run. The first assay consisted of an oligonucleotide microarray experiment in which amine-modified and unmodified 40mers were printed onto the amine-reactive coated slides. This assay provided an assessment of NHS activity of the films, with the unmodified oligo serving as an internal non-specific binding control. Details of the oligonucleotide microarray experiment are provided in the Supporting Information. Briefly amine-modified and unmodified $40 \mathrm{mer}$ probes with identical sequences were printed to the three slides per batch using a PerkinElmer Piezorray non-contact arrayer. After arraying, the slides were incubated for 1 hour at $50 \%$ humidity and room temperature $\left(\sim 22^{\circ} \mathrm{C}\right)$ and then deactivated as described in the "Surface Deactivation" section above. Dye-labeled complementary 40mer target was hybridized for one hour at $42^{\circ} \mathrm{C}$ using Whatman S\&S FastFrame chambers. Processed arrays were scanned on a Tecan LS-400 laser scanner. Additional details (buffers, reagent concentrations, times, etc.) are provided in the Supporting Information.

As a second assay to test for batch-to-batch variability, the same slides used in the oligo microarray experiment were also checked for protein non-specific binding using $10 \%$ fluorescently labeled goat serum as described above. During the oligo hybridization, seperate FastFrame chambers were applied to the slides to allow regions to be incubated with the labeled serum. A bare glass slide was used as the positive control in the serum assay.

\section{RESULTS AND DISCUSSION}

\section{Surface Analysis}

AFM and Ellipsometry-Representative AFM results are presented in Figure 2. Additional AFM data are provided in the Supporting Information, including topography and phase plots for dry and wet coatings on glass (Figure S-1) and on ITO (Figure S-2). Figure 2(a) shows that the dry, coated film is very smooth (peak-to-trough roughness of less than $1 \mathrm{~nm}$, RMS roughness $=0.29 \mathrm{~nm}$ ), comparable to the underlying glass substrate. To probe film thickness, the AFM tip was driven into the film and dragged in contact mode to form a scratch (data not shown). Scratch analysis shows a dry film thickness of approximately $20 \mathrm{~nm}$.

Upon hydration, film topography and thickness change significantly, and sequential scans were used to qualitatively monitor film hydration kinetics. Underwater scans (Figs. 2(b-d)) show a small hole defect visible on the right side of the field of view. Line profiles across this defect provide an assessment of thickness and topography evolution: at the 10-minute time point, the hole is on the order of $60 \mathrm{~nm}$ deep, suggesting that the film has swollen significantly.

Subsequent scans over this same location show that the hole largely "heals" over time, presumably from polymer hydration and expansion. Hydrated films reach a stable AFM surface topography after approximately 30 minutes of water exposure, achieving a final peak-to-trough surface roughness of approximately 10 to $20 \mathrm{~nm}$ (RMS roughness $=3.20 \mathrm{~nm}$ ). Based on these AFM scratch and hole measurements, dry film thickness on glass is approx. $10-20 \mathrm{~nm}$, while the hydrated film thickness is approx. $50-100 \mathrm{~nm}$ with a known peak-to-trough surface roughness of $10-20 \mathrm{~nm}$.

AFM results for polymer-coated ITO surfaces are qualitatively similar to those on the coated glass samples, with the primary difference being the more pronounced film domain structure on ITO (see Supplemental Fig. S-2). It will be shown below that ITO versus glass differences 
also appear in the XPS data. Despite the physical differences on the two substrates, however, non-specific binding properties of coating on both substrates are very similar.

AFM results show polymer film thickness and hydration behavior similar to PEG films based on adsorbed amphiphiles with intermediate PEG molecular weights (2000 to 8000$)^{20}$ and poly (AAm-co-EG) interpenetrating networks. ${ }^{16}$ The polymer film is thicker than the more common grafted PEGs, PEG brushes, and SAMs, which have dry film thickness ranging from approximately 1 to $5 \mathrm{~nm}^{10,12,15}$ depending on molecular weight and coverage.

Spectroscopic ellipsometry data for polymer-coated $\mathrm{SiO}_{2} / \mathrm{Si}$ wafers provide dry film thicknesses complementing information generated with AFM. Cauchy model fits were used to estimate polymer thicknesses and optical constants. Polymer thickness was measured at multiple locations on three different wafer substrates, with average dry film thickness of $9.1 \pm$ $0.2 \mathrm{~nm}$. The $\sim 9 \mathrm{~nm}$ thickness result corroborates the AFM conclusion that the crosslinked film is thicker than grafted PEGs and SAMs.

XPS-XPS was used to analyze the NHS-activated polymer-coated glass and ITO surfaces, and examine the effects of curing the coating at $100^{\circ} \mathrm{C}$. Table 1 provides compositional XPS data. Additional high resolution XPS species data are provided in Supporting Table S-1. XPSmeasured surface composition for polymer-coated glass slides shows excellent agreement with the theoretical composition calculated from the input chemical formulation. The carbon:oxygen atomic ratio measured for the polymer film is 2.5 , a value consistent with a PEG film enriched with additional carbon, likely from alkyl components of the azidosilane crosslinker and PST matrix-forming molecules. Complete attenuation of glass substrate XPS peaks confirms a relatively thick coating ( $\sim 9$ to $20 \mathrm{~nm}$ based on ellipsometry and AFM). We note here that based on high-resolution Si2p data (Table S-2), the difference between the theoretical and measured Si2p signal on glass is not due to substrate detection (i.e., not amorphous silicon oxide), since on glass the Si2p peak (amorphous Si-O) had a binding energy of $103 \mathrm{eV}$, while XPS binding energy for the Si2p peak in the polymer coating was at 102.4 $\mathrm{eV}$, a value more consistent with a silane $\mathrm{Si}-\mathrm{O}$ peak.

Elemental composition of the polymer film on ITO is similar to that measured on glass. Indium and tin peaks from the substrate, however, are not completely attenuated, suggesting that the coating on ITO is not as thick as that on glass ( $<5 \mathrm{~nm}$ based on XPS sampling depth). This assertion is supported by the $\mathrm{C} / \mathrm{O}$ ratio of 2.0, suggesting that oxygen from the substrate (ITO) is detected. Presumably, the underlying surface energy or physical features of the ITO affect the polymer coating. The slight differences in the XPS results for ITO versus glass are consistent with the AFM results discussed above.

The combination of AFM, ellipsometry, and XPS provide a reasonably complete picture of film thickness. The dry film is $\sim 9 \mathrm{~nm}$ thick on $\mathrm{SiO}_{2} / \mathrm{Si}$ (ellipsometry), approximately 8 to 20 $\mathrm{nm}$ thick on glass (XPS \& AFM) and 3 to $5 \mathrm{~nm}$ on ITO (XPS). The varying thickness for the different substrates is not unexpected, given that spin-coat film thickness depends in part on the surface energy of the substrate. Upon hydration, the films expand significantly to thicknesses of 50 to $100 \mathrm{~nm}$.

Cure Analysis-High-resolution XPS and FTIR spectra were used to gain insight into the chemical evolution of the azidosilane cross-linker. Detailed XPS and FTIR results and discussion are provided in the "Polymer Cure Study" section of the Supporting Information. Results presented there are consistent with a fractional conversion of the azide to nitrene with subsequent insertion into aliphatic carbon chains in the coating, suggesting the desired crosslinking reactions indeed occur. 
Contact Angle-Clean, uncoated glass and ITO substrates exhibited water contact angles less than $10^{\circ}$. Uncoated TCPS dishes had contact angles of approximately $57^{\circ}$, consistent with literature values ${ }^{45}$. Static contact angles of the NHS-activated coatings were: $62.0^{\circ} \pm 1.5^{\circ}$ for glass, $54.4^{\circ} \pm 2.1^{\circ}$ for ITO, and $60.7^{\circ} \pm 0.6^{\circ}$ for TCPS. Deactivation with methoxyethylamine results in contact angles of $49.6^{\circ} \pm 3.4^{\circ}$ for glass, $46.7^{\circ} \pm 3.3^{\circ}$ for ITO, and $59.2^{\circ} \pm 0.7^{\circ}$ for TCPS. Average intra-slide (6-spot) coefficients of variation were less than $6 \%$ for all substrate types.

These coatings are less hydrophilic than the most common PEG-based hydrogel chemistries, which typically show water contact angles between $30^{\circ}$ and $40^{\circ} 15,16,25,46-48$. The decrease upon deactivation is the result of removing the NHS leaving group and replacing it with a mixture of terminal methoxy and carboxyl groups (the latter due to competing hydrolysis reactions). The slightly lower contact angles for coated ITO correlate with the AFM results (Figure S-2) showing an altered surface microstructure on this substrate.

Dynamic water contact angles measured on polymer-coated glass slides $(n=6)$ showed advancing $\left(68.7^{\circ} \pm 0.7^{\circ}\right)$ and receding $\left(14.8^{\circ} \pm 2.0^{\circ}\right)$ angles with a hysteresis of $53.9^{\circ}$. This is a large, but not unprecedented hysteresis. Observed hysteresis is attributed to large PEG tethered chain mobilities ${ }^{49}$ that respond to the moving three-phase line, and is not ascribed to surface topological effects. An alternative explanation for large hysteresis would be release of surface-active components from the film into the test droplet during the measurement (e.g. leaching). This was ruled out by comparing droplet surface tension before and after exposure to the polymer surface. Large hysteresis is not uncommon for swellable hydrogels 50,51 , but this behavior is different from SAMs or grafted PEG brushes where the hysteresis is smaller, typically on the order of $9^{\circ}$ to $15^{\circ} 15,25,48$.

Protein Non-Specific Binding-Non-specific protein binding results for fluorescent assays on coated glass slides are presented in Figure 3. Results show that the polymer film provides significant inhibition of serum-based NSB. For $10 \%$ serum, the polymer provides $97 \%$ reduction in non-specifically adsorbed serum components, with even better percent reductions in more dilute serum. This result suggests that the polymer coating is particularly good at inhibiting adsorption of the primary albumin and globulin protein components that comprise the largest protein fractions ( $>90 \%$ ) in serum. The coating provides a $90 \%$ reduction in fibrinogen NSB relative to bare glass control at $100 \mathrm{micrograms} / \mathrm{ml}$ fibrinogen, although the relative reduction was not as good for $10 \mathrm{micrograms} / \mathrm{ml}$ fibrinogen (73\% reduction). Lysozyme NSB was also significantly inhibited. At 100 microgram/ml lysozyme, the coating provided greater than $95 \%$ reduction in NSB relative to bare glass. For all three protein systems (serum, fibrinogen, lysozyme), the polymer-coated glass slides out-performed BSA-blocked glass slides.

Figures 3(d) and (e) show fibrinogen NSB on ITO- and TCPS-coated surfaces measured by enzyme immunoassay. Reduction of fibrinogen NSB was similar to that seen on coated glass. Performance of the polymer-coated ITO and TCPS surfaces was comparable to BSA-blocking, suggesting that BSA-blocking works better on ITO and TCPS than it does on glass, an expected result given that BSA blocking is best on surfaces that present hydrophobic surface sites ${ }^{1}$. Note also that the $0 \mathrm{microgram} / \mathrm{ml}$ fibrinogen control wells yield relatively strong NSB signals on the bare ITO and TCPS surfaces, a result of non-specific binding of anti-fibrinogen antibody to these surfaces. The polymer coating completely inhibits this effect without the use of special blocking reagents in the diluent buffer.

Inhibition of Bacterial Adhesion-Bacterial retention assay results are presented in Figure 4. S. aureus adhesion to bare glass and bare ITO surfaces is strong and largely irreversible after a one hour static incubation in the flow cell. Even at a wall shear rate of $270 \mathrm{sec}^{-1}$ (data not 
shown), $>60 \%$ of the $S$. aureus microorganisms remained bound to the glass surface, and $>$ $80 \%$ remain bound to bare ITO. In contrast, essentially all S. aureus cells were removed from polymer-coated surfaces at the lowest shear rate, suggesting that adhesion to the polymer surface is weak in the time frame tested. Results for K. pneumoniae were similar. $K$. pneumoniae is less adherent to bare glass than $S$. aureus, consistent with the fact that different bacterial strains and species exhibit widely different adhesive properties ${ }^{38}$. K. pnuemoniae cells were completely removed from the polymer coated surfaces at the lowest shear rate (4.6 $\left.\mathrm{sec}^{-1}\right)$. For these representative strains, performance is qualitatively comparable to that seen on ethylene oxide-terminated SAMs ${ }^{27}$ and long chain-length PEG brushes ${ }^{52}$. It is important to reiterate that these data are for short-term bacterial exposure ( 1 to 2 hours). This time frame was selected because it is particularly relevant to in vitro bioassays. Additional bacterial adhesion studies, including 7 day coating stability assessment in biological fluids, are published elsewhere 53 .

Mammalian Cell Adhesion-Figure 5 presents NIH 3 T3 fibroblast 24-hour adhesion data from $10 \%$ serum-containing media to the inert (i.e., hydrolyzed) polymer coating, GRGDSmodified-polymer coating, and the TCPS positive control. Fig. 5(a) shows virtually no fibroblast adhesion to the hydrolyzed polymer-coated surface in the 24-hour culture test, a result consistent with the inhibition of protein and bacterial adhesion presented already. The RGD- functionalized coating, however, showed significant NIH 3T3 cell attachment, including greater attachment than even the TCPS control (Fig. 5(b) and (c)). Fig. 5(d) provides a quantitative analysis of cell attachment for the three surfaces. This last plot shows that the nonfouling surface chemistry is readily functionalized to actively promote specific adhesion.

Reproducibility Assays-Figure 6(a) and (b) present results for the oligonucleotide microarray assay. Reproducibility is reported as percent coefficient of variance $(\% \mathrm{CV})$, defined $\% \mathrm{CV}=100 *$ (StDev/Mean). Intra-batch reproducibility of amine-reactivity as measured by the amine-modified oligo spot signal (10 spot subarrays, two subarrays per slide, three slides per batch; 60 spot statistics) was 5.4\%, 5.2\%, and $2.0 \% \mathrm{CV}$ for the three batches tested. Interbatch reproducibility was $4.1 \% \mathrm{CV}$ for the three batches assayed here. Specificity of the NHS to amine-oligo is demonstrated by the very low signal seen on the unmodified-oligo probe spots.

The serum assay data show quantitative results very similar to those presented in Figure 3, with the coated slides giving $<1 \%$ of the non-specific binding signal seen on the bare glass controls. Reproducibility of the serum non-specific binding signal for the three batches tested was $7.2 \%$ $\mathrm{CV}$.

Regarding reproducibility, we also note here that this formulation has been used in over 400 quality controlled production batches constituting more than 45,000 coated substrates distributed worldwide for microarray applications.

\section{CONCLUSIONS}

A novel, multi-component, cross-linked polymer surface chemistry that effectively inhibits the non-specific adsorption of proteins, bacteria, and mammalian cells has been described and characterized. The coating is unique in the context of poly(ethylene glycol) surface chemistries in that it comprises a simple, commercially viable, single-step coating process applicable to a variety of solid substrate materials. Further, the polymer surface chemistry can be modified in situ to provide specific attachment chemistries within the same low non-specific binding coating matrix. The coating technology is particularly relevant for in vitro bioassay applications where the combination of low non-specific binding and highly specific immobilization is 
required for assay sensitivity. The long-term stability of this coating in biological milieu is the subject of parallel studies ${ }^{53}$.

\section{Supplementary Material}

Refer to Web version on PubMed Central for supplementary material.

\section{Acknowledgements}

We thank Dr. G. Mao for technical advice regarding the coating formulation chemistry, Y-F. Lu and Dr. B. Parkinson, Colorado State University (CSU), for assistance with AFM measurements, Dr. E. Fisher (CSU) for use of the dynamic contact angle tool, and Dr. S. Kohli and D. Heyse (CSU CIF) for assistance with ellipsometry measurements.

\section{References}

1. Butler JE. Methods 2000;22:4-23. [PubMed: 11020313]

2. Kusnezow W, Hoheisel JD. J Mol Recognit 2003;16:165-176. [PubMed: 12898667]

3. Lang P, Yeow K, Nichols A, Scheer A. Nat Rev Drug Discovery 2006;5:343-356.

4. Harris, JM., editor. Poly(ethylene Glycol) Chemistry: Biotechnical and Biomedical Applications. Plenum Press; New York: 1992.

5. Hoffman AS. J Biomat Sci, Polym Ed 1999;10:1011-1014.

6. Leckband D, Sheth S, Halperin A. J Biomat Sci, Polym Ed 1999;10:1125-1147.

7. Ostuni E, Chapman RG, Holmlin RE, Takayama S, Whitesides GM. Langmuir 2001;17:5605-5620.

8. Gombotz WR, Wang GH, Horbett TA, Hoffman AS. J Biomed Mater Res 1991;25:1547-1562. [PubMed: 1839026]

9. Mao G, Castner DG, Grainger DW. Chem Mater 1997;9:1741-1750.

10. Sofia SJ, Premnath V, Merrill EW. Macromolecules 1998;31:5059-5070. [PubMed: 9680446]

11. Emoto K, Van Alstine JM, Harris JM. Langmuir 1998;14:2722-2729.

12. Lee SW, Laibinis PE. Biomaterials 1998;19:1669-1675. [PubMed: 9840002]

13. Jo S, Park K. Biomaterials 2000;21:605-616. [PubMed: 10701461]

14. Xia N, Hu Y, Grainger DW, Castner DG. Langmuir 2002;18:3255-3262.

15. Schlapak R, Pammer P, Armitage D, Zhu R, Hinterdorfer P, Vaupel M, Fruhwirth T, Howorka S. Langmuir 2006;22:277-285. [PubMed: 16378432]

16. Bearinger JP, Castner DG, Golledge SL, Rezania A, Hubchak S, Healy KE. Langmuir 1997;13:51755183.

17. Dalsin JL, Lin L, Tosatti S, Voros J, Textor M, Messersmith PB. Langmuir 2005;21:640-646. [PubMed: 15641834]

18. Wazawa T, Ishizuka-Katsura Y, Nishikawa S, Iwane AH, Aoyama S. Anal Chem 2006;78:25492556. [PubMed: 16615763]

19. Lee JH, Kopecek J, Andrade JD. J Biomed Mater Res 1989;23:351-368. [PubMed: 2715159]

20. Malmsten M, Van Alstine JM. J Colloid Interface Sci 1996;177:502-512.

21. Li JT, Carlsson J, Lin JN, Caldwell KD. Bioconjug Chem 1996;7:592-599. [PubMed: 8889022]

22. Kenausis GL, Voeroes J, Elbert DL, Huang N, Hofer R, Ruiz-Taylor L, Textor M, Hubbell JA, Spencer ND. J Phys Chem B 2000;104:3298-3309.

23. Ruiz-Taylor LA, Martin TL, Zaugg FG, Witte K, Indermuhle P, Nock S, Wagner P. Proc Natl Acad Sci USA 2001;98:852-857. [PubMed: 11158560]

24. Huang NP, Michel R, Voros J, Textor M, Hofer R, Rossi A, Elbert DL, Hubbell JA, Spencer ND. Langmuir 2001;17:489-498.

25. Prime KL, Whitesides GM. J Am Chem Soc 1993;115:10714-10721.

26. Mrksich M, Whitesides GM. ACS Symposium Series 1997;680:361-373.

27. Ostuni E, Chapman RG, Liang MN, Meluleni G, Pier G, Ingber DE, Whitesides GM. Langmuir 2001;17:6336-6343. 
28. Johnston EE, Bryers JD, Ratner BD. Langmuir 2005;21:870-881. [PubMed: 15667162]

29. Mao, G.; Metzger, S.; Lochhead, MJ. United States Patents. 6,844,028 and 7,067,194.

30. Gonzalez L, Rodriguez A, De Benito JL, Marcos-Fernandez A. J Appl Polym Sci 1997;63:13531359.

31. Scriven, EFV., editor. Azides and Nitrenes. Academic Press; New York: 1984.

32. Gong P, Harbers GM, Grainger DW. Anal Chem 2006;78:2342-2351. [PubMed: 16579618]

33. Horbett TA, Weathersby PK. J Biomed Mater Res 1981;15:403-423. [PubMed: 7348274]

34. Bergers JJ, Vingerhoeds MH, van Bloois L, Herron JN, Janssen LH, Fischer MJ, Crommelin DJ. Biochemistry 1993;32:4641-4649. [PubMed: 8485142]

35. Unsworth LD, Sheardown H, Brash JL. Langmuir 2005;21:1036-1041. [PubMed: 15667186]

36. Bergstrom K, Holmberg K, Safranj A, Hoffman AS, Edgell MJ, Kozlowski A, Hovanes BA, Harris JM. J Biomed Mater Res 1992;26:779-790. [PubMed: 1527100]

37. Model MA, Healy KE. J Biomed Mater Res 2000;50:90-96. [PubMed: 10644968]

38. Busscher HJ, van der Mei HC. Clin Microbiol Rev 2006;19:127-141. [PubMed: 16418527]

39. Li N, Tourovskaia A, Folch A. Crit Rev Biomed Eng 2003;31:423-488. [PubMed: 15139302]

40. Drumheller PD, Hubbell JA. Anal Biochem 1994;222:380-388. [PubMed: 7864362]

41. Harbers GM, Healy KE. J Biomed Mater Res, Part A 2005;75:855-869.

42. Hersel U, Dahmen C, Kessler H. Biomaterials 2003;24:4385-4415. [PubMed: 12922151]

43. Pierschbacher MD, Ruoslahti E. Nature 1984;309:30-33. [PubMed: 6325925]

44. Godek ML, Duchsherer NL, McElwee Q, Grainger DW. Biomed Sci Instrum 2004;40:7-12. [PubMed: 15133927]

45. Koenig AL, Gambillara V, Grainger DW. J Biomed Mater Res 2003;64:20-37.

46. Harbers GM, Gamble LJ, Irwin EF, Castner DG, Healy KE. Langmuir 2005;21:8374-8384. [PubMed: 16114945]

47. Herrwerth S, Eck W, Reinhardt S, Grunze M. J Am Chem Soc 2003;125:9359-9366. [PubMed: 12889964]

48. Pale-Grosdemange C, Simon ES, Prime KL, Whitesides GM. J Am Chem Soc 1991;113:12-20.

49. Karlsson JO, Gatenholm P. Macromolecules 1999;32:7594-7598.

50. Andrade, JD., editor. Surface and Interfacial Aspects of Biomedical Polymers. Plenum Press; New York: 1985.

51. Emoto K, Nagasaki Y, Kataoka K. Langmuir 1999;15:5212-5218.

52. Roosjen A, Boks NP, Van der Mei HC, Busscher HJ, Norde W. Colloids Surf, B 2005;46:1-6.

53. Saldiagarra Fernandez IC, van der Mei HC, Lochhead MJ, Grainger DW, Busscher HJ. Biomaterials. 2007in press 


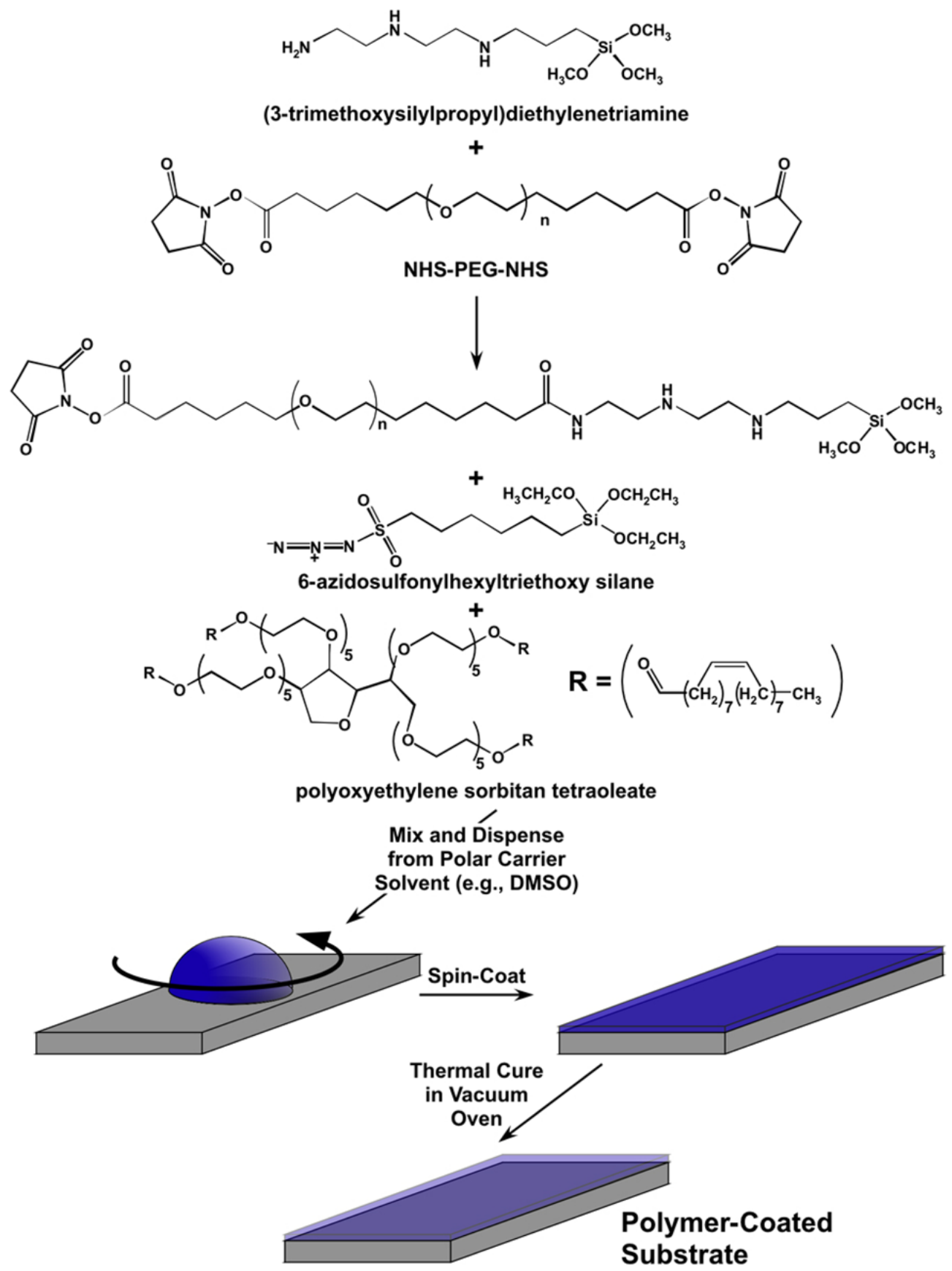

Figure 1.

Polymer coating components and process schematic. 

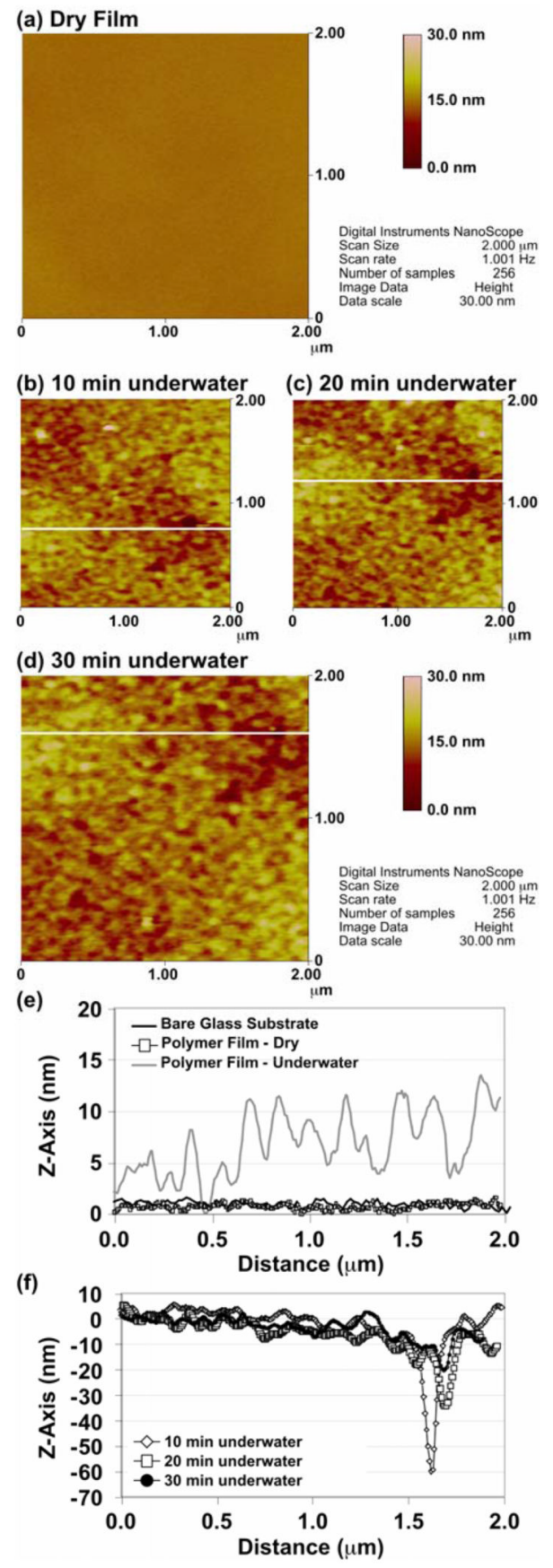

Figure 2.

(a) Representative tapping mode AFM topography scan of a dry, polymer-coated glass slide. (b-d) Underwater tapping mode scans after 10, 20, and 30 minutes of water exposure. (e) Representative AFM line profiles for the bare glass substrate, the dry polymer film, and the hydrated polymer film. (f) Sequence of line profiles showing hydration of the polymer film. These profiles correspond to the lines on images $(b-d)$. Note that the image drifts slightly during acquisition of the underwater scans. 
(a)

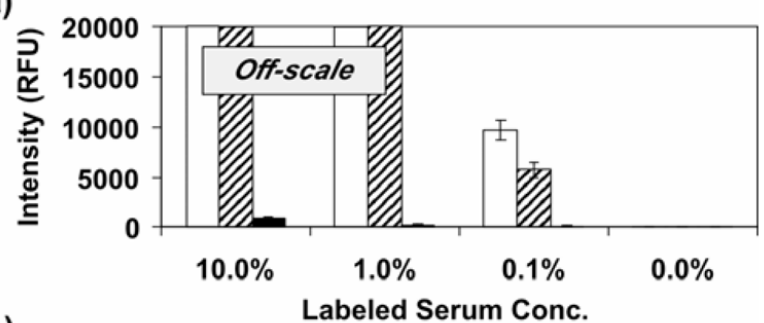

(b)

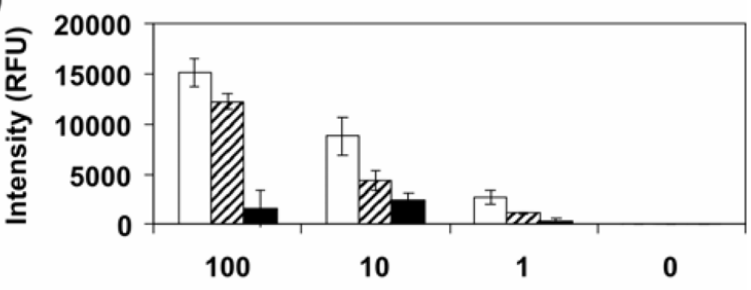

(c)

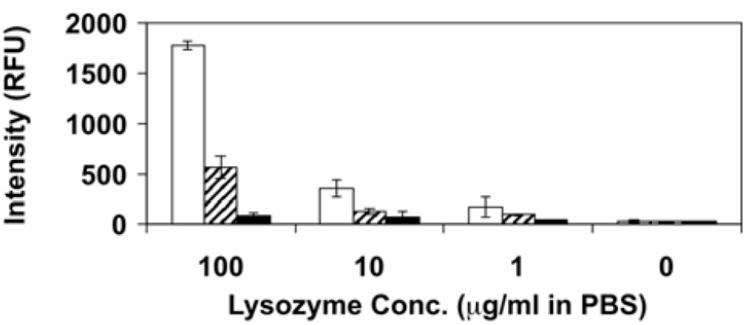

(d)

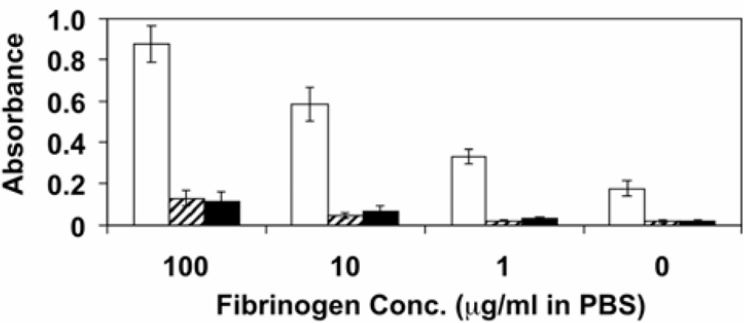

(e)

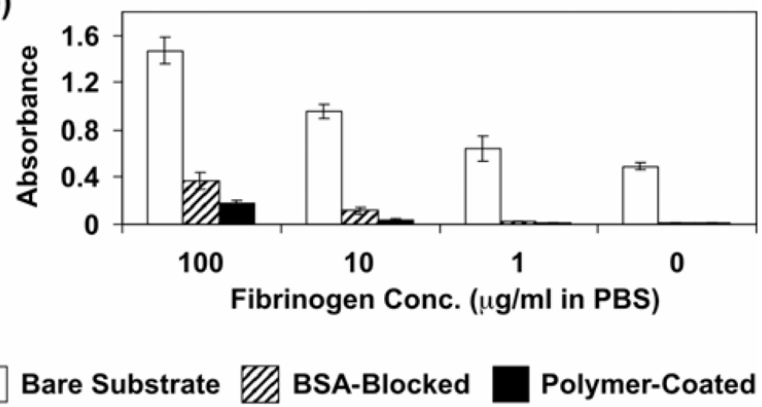

Figure 3.

$(a-c)$ Fluorescently labeled protein non-specific binding on uncoated, BSA-blocked, and polymer-coated glass slides. (a) Dye-labeled serum non-specific binding. The bars are off-scale for the bare and BSA-blocked glass at $10 \%$ and $1 \%$ serum. Fluorescence intensity values for bare glass are 32,293 and 28,776 RFU (10\% and 1\% serum, respectively), and 39,132 and 29,103 RFU for BSA-blocked glass. (b) Human fibrinogen and (c) lysozyme non-specific binding on glass slides using the fluorescent half-sandwich assays. For $(\mathrm{a}-\mathrm{c}), \mathrm{RFU}=$ relative fluorescence units as generated by the Tecan laser scanner on the green $(532 \mathrm{~nm})$ channel at 55\% PMT; values reported for each concentration are three slide averages, with error bars representing one interslide standard deviation. (d) Human fibrinogen non-specific binding to 
ITO and (e) TCPS as measured by enzyme immunoassay. Absorbance units are optical density at $450 \mathrm{~nm}$. Values reported for each concentration are three slide averages, three wells per slide ( 9 wells total), with error bars representing one standard deviation. 

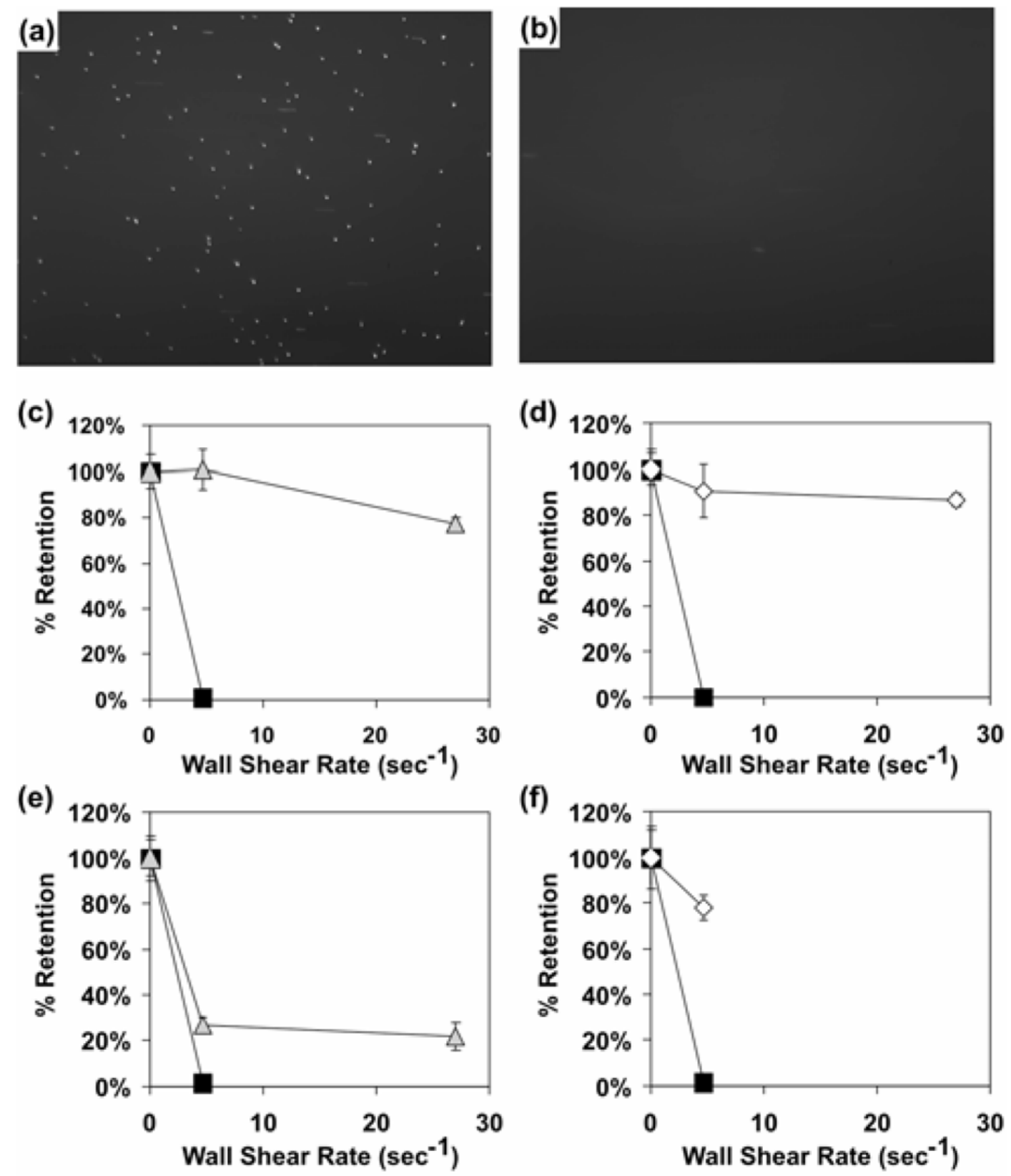

\section{Bare Glass $\diamond$ Bare ITO Polymer-Coated}

Figure 4.

Bacterial adhesion to bare and polymer-coated surfaces in a microfluidic flow cell. (a) Representative image of a bare glass flow cell surface after $S$. aureus exposure and rinse at a shear rate of $4.6 \mathrm{sec}^{-1}$. (b) Image of polymer-coated surface under same conditions as (a). Streaks in the images are bacteria moving laterally during the image exposure (not counted in the analysis). (c) Percent retention of $S$. aureus on bare and polymer-coated glass flow cell surfaces at different rinse wall shear rates. (d) S. aureus retention on ITO. (e) K. pneumoniae retention on glass. (f) $K$. pneumoniae on ITO. Values are normalized cell counts averaged over five microscope fields of view. Error bars represent one standard deviation. 

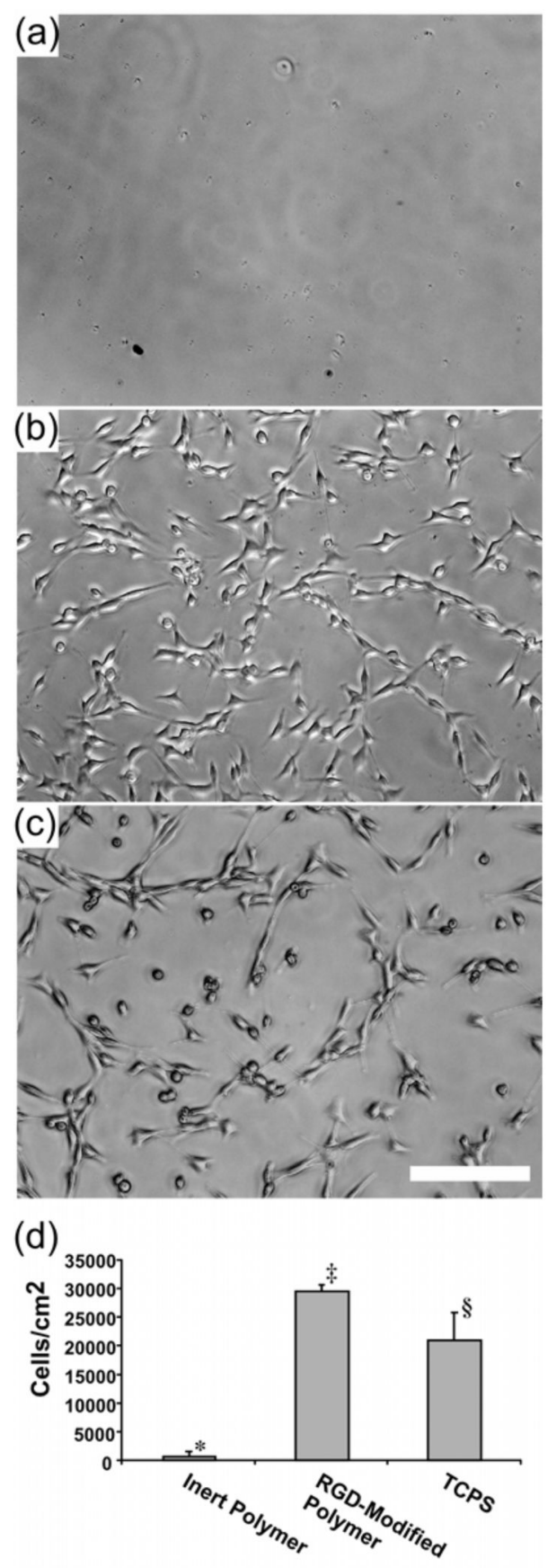

Figure 5.

NIH 3T3 fibroblast adhesion $(24 \mathrm{~h})$ to the inert polymer surface, GRGDS-modified polymer surface, and TCPS in 10\% serum-containing media. Representative images for each surface: (a) hydrolyzed "inert" polymer coating; (b) GRGDS-modified polymer; and (c) TCPS positive control. (d) Total cell number determined using digital image analysis; surfaces not in the same group $(*, \S, \ddagger)$ were statistically different from one another $(\mathrm{p}<0.05$; ANOVA with Tukey Kramer post-hoc test). Scale bar $=200$ micrometers. 
(a)

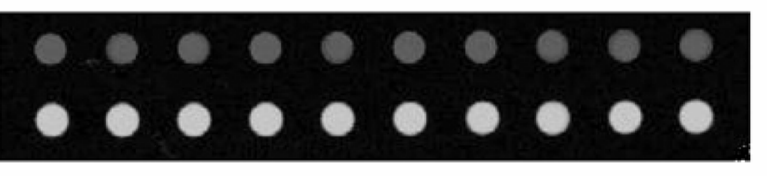

(b)

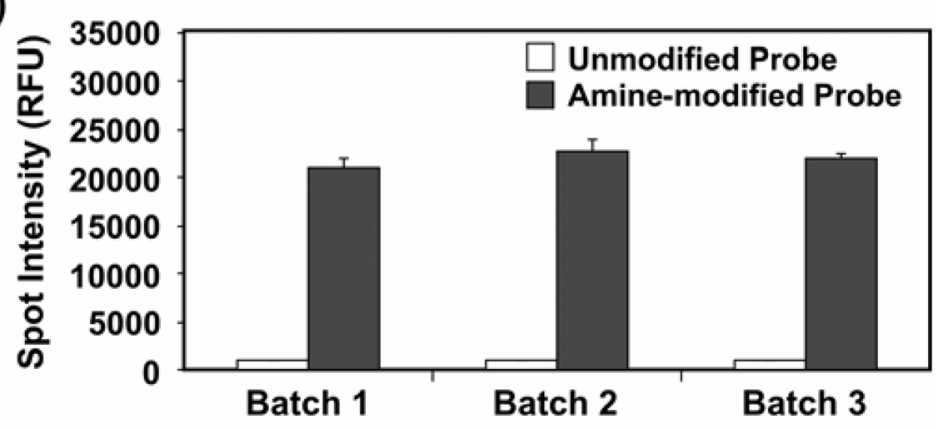

(c)

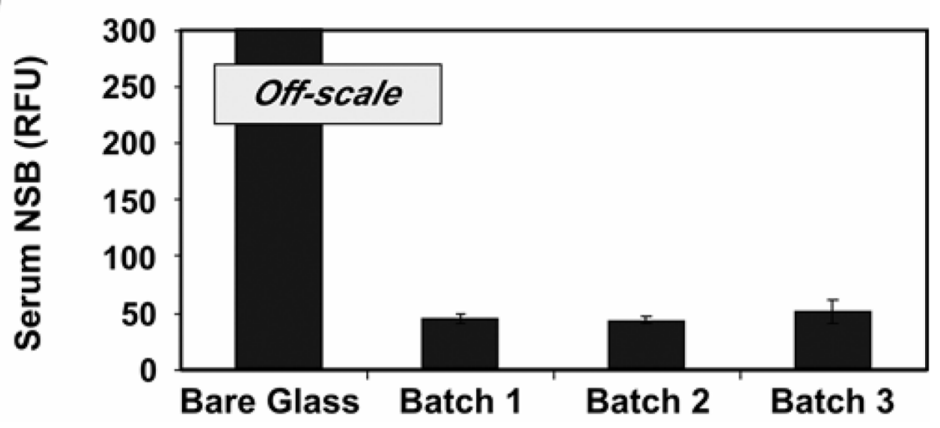

Figure 6.

Batch-to-batch reproducibility data. (a) Representative microarray image of unmodified (top row) and amine-modified (bottom row) oligos printed onto the polymer coating and then hybridized with Cy3-labeled oligo target. (b) Oligo microarray assay results and (c) dye labeled serum non-specific binding results for three representative production batches prepared over a one month period. Three slides per batch were assayed; each bar represents a three slide mean and the error bars represent $+/-$ one standard deviation. Signal for the bare glass control in the serum assay is off-scale at 18,701 RFU. (RFU = relative fluorescence units). 


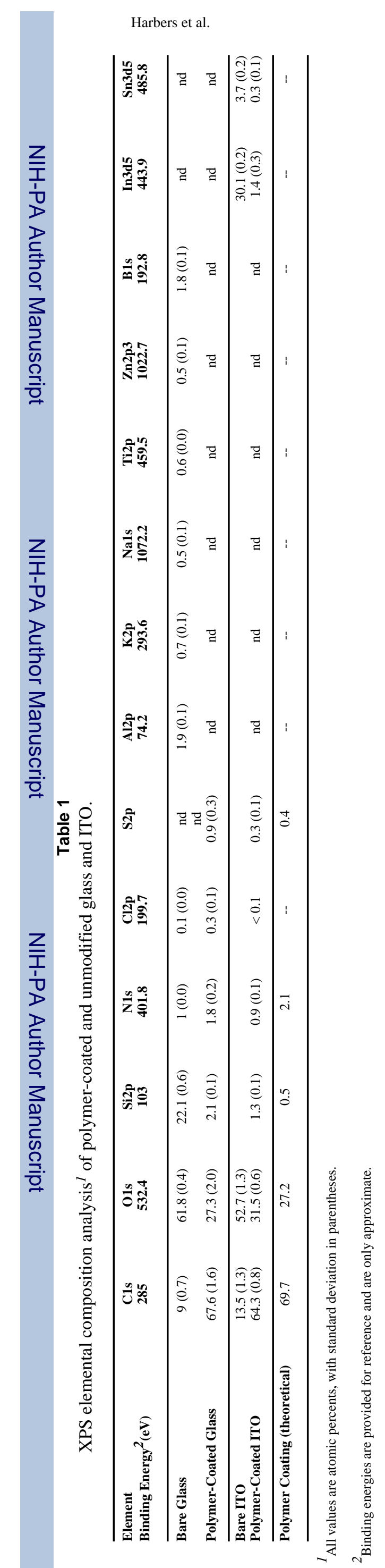

